\title{
Potential Implications of Quercetin in Autoimmune Diseases
}

\author{
Pan Shen ${ }^{1}$, Weiji Lin ${ }^{1}$, Xuan Deng ${ }^{2}$, Xin Ba ${ }^{1}$, Liang $\operatorname{Han}^{1}$, Zhe Chen ${ }^{1}$, Kai Qin ${ }^{1}$, \\ Ying Huang ${ }^{1}$ and Shenghao $\mathrm{Tu}^{1 *}$
}

${ }^{1}$ Department of Integrated Chinese Traditional and Western Medicine, Tongji Hospital, Tongji Medical College of Huazhong University of Science and Technology, Wuhan, China, ${ }^{2}$ Department of Nephrology, Zhongnan Hospital of Wuhan University, Wuhan, China

OPEN ACCESS

Edited by:

Narendra Prasad Singh, University of South Carolina,

United States

Reviewed by:

José María Pego Reigosa,

University Hospital Complex

of Vigo, Spain

Bin Li,

Shanghai Jiao Tong University, China

${ }^{*}$ Correspondence:

Shenghao Tu

shtu@tjh.tjmu.edu.cn

Specialty section:

This article was submitted to

Autoimmune and

Autoinflammatory Disorders,

a section of the journal

Frontiers in Immunology

Received: 31 March 2021

Accepted: 10 May 2021

Published: 23 June 2021

Citation:

Shen P, Lin W, Deng X, Ba X, Han L,

Chen Z, Qin K, Huang Y and Tu S

(2021) Potential Implications of

Quercetin in Autoimmune Diseases.

Front. Immunol. 12:689044.

doi: 10.3389/fimmu.2021.689044
Autoimmune diseases are a worldwide health problem with growing rates of morbidity, and are characterized by breakdown and dysregulation of the immune system. Although their etiology and pathogenesis remain unclear, the application of dietary supplements is gradually increasing in patients with autoimmune diseases, mainly due to their positive effects, relatively safety, and low cost. Quercetin is a natural flavonoid that is widely present in fruits, herbs, and vegetables. It has been shown to have a wide range of beneficial effects and biological activities, including anti-inflammation, anti-oxidation, and neuroprotection. In several recent studies quercetin has reportedly attenuated rheumatoid arthritis, inflammatory bowel disease, multiple sclerosis, and systemic lupus erythematosus in humans or animal models. This review summarizes the evidence for the pharmacological application of quercetin for autoimmune diseases, which supports the view that quercetin may be useful for their prevention and treatment.

Keywords: autoimmune diseases, quercetin, rheumatoid arthritis, inflammation, oxidative stress

\section{INTRODUCTION}

Autoimmune diseases are a heterogeneous cluster of disorders characterized by systemic syndromes, in which autoreactive adaptive immune responses contribute to immune-mediated damage to cells and organs (1). The pathogenesis and etiology of these diseases is not completely clear, but a complex interplay of genetic risk, environmental factors, mental factors, and stochastic events is considered to disrupt the balance of immunological tolerance and autoimmunity $(2,3)$. The polyphenol quercetin $\left(3,3^{\prime}, 4^{\prime}, 5,7\right.$-pentahydroxyflavone) is a dietary flavonoid that is commonly found in vegetables (asparagus), fruits (apples, capers, chokeberries, cranberries), and medicinal herbs including lovage, dill, cilantro, and radish leaves (4-6). Many previous studies have shown that appropriate doses of quercetin have a variety of biological activities and neuroprotective, antiallergic, anti-oxidant, anti-inflammatory, immunomodulatory, anti-microbial, and anti-tumor effects (7-12). Various ongoing scientific studies are investigating the use of natural products to develop drugs to treat autoimmune diseases. Quercetin may be useful as a dietary supplement to prevent or treat autoimmune diseases.

In the present manuscript we will summarize the protective effects of quercetin in multiple autoimmune diseases, and evaluate the molecular mechanisms of quercetin that can modulate immune responses. 


\section{BIOLOGICAL ACTIVITY AND FUNCTIONS OF QUERCETIN}

In dietary supplements, daily doses of quercetin are usually in the range of 1 to $250 \mathrm{mg}$ (13). After ingestion quercetin can combine with salivary proteins to form soluble protein-quercetin binary aggregates (14). Upon arrival in the small intestine quercetin is deglycosylated by lactate phlorizin hydrolase to produce quercetin aglycon. In the cecum and colon, quercetin glucosides can be directly taken in via sodium-dependent glucose transporter-1, or secreted into the lumen via multidrug resistance protein 2 (15). Quercetin can be absorbed into epithelial cells via lipophilicity-dependent dispersion. Before entering the circulatory system most of the quercetin ingested from the intestinal cavity is converted into conjugated metabolites (16). Approximately $60 \%-81 \%$ of quercetin is channeled to the liver via the epithelium, where it is metabolized and converted into a bioavailable form (17). Most quercetin and its metabolites are excreted via the intestines, but small amounts are excreted by the kidneys in the urine (18). Recent studies suggest that gut microbiota participate in the production of glycosidases and enzymes that convert quercetin into more readily absorbable molecules $(19,20)$. Quercetin can evidently be metabolized as glucuronidated, methylated, and sulphated derivatives such as homoprocatechuic acid, protocatechuic acid, and 4-hydroxybenzoic acid (21).

Quercetin reportedly exerts powerful anti-inflammatory effects, mainly via inhibition of cytokine production, reduction of cyclooxygenase and lipoxygenase expression, and maintaining the stability of mast cells (22-24). It can reportedly reduce Streptococcus suis-induced inflammation by inhibiting the activation of p38 mitogen-activated protein kinase, extracellular signal-related kinases (ERK1/2), and nuclear factor kappa B (NF$\mathrm{\kappa B})$. It can also reduce the production of pro-inflammatory cytokines such as tumor necrosis factor (TNF)- $\alpha$, interleukin (IL)-1 $\beta$, and IL-6 (25). In one study, treatment with quercetin at a dose of $25 \mathrm{mg} / \mathrm{kg}$ in diabetic rats reduced the production of prostaglandin E-2, IL-1 $\beta$, and leukotriene B-4, promoting wound healing in rats (26). Similarly quercetin inhibited the production of TNF- $\alpha$, IL-6, and IL-1 in lipopolysaccharide-activated human mononuclear U937 cells (27). An indomethacin-mediated increase in myeloperoxidase activity in stomach and ileum tissues, as well as activation of NF- $\mathrm{KB}$ and the production of IL8 in Caco-2 cells were also inhibited by quercetin $(10 \mu \mathrm{g} / \mathrm{mL})(28)$. In lipopolysaccharide-treated RAW264.7 cells, quercetin inhibited the production of TNF- $\alpha$ and nitric oxide synthase (iNOS), and the phosphorylation and activation of Jun $\mathrm{N}$-terminal kinase/ stress-activated protein kinase. Additionally, quercetin suppressed NF- $\kappa \mathrm{B}$ translocation, AP-1, and NF- $\mathrm{KB}-\mathrm{DNA}$-binding and reporter gene transcription (29). It can reportedly protect human umbilical vein endothelial cells from inflammation induced by $\mathrm{H}_{2} \mathrm{O}_{2}$, which is mediated by downregulation of vascular cell adhesion molecule 1 and CD80 (30). As well as inhibiting pro-inflammatory cytokines such as TNF- $\alpha$, IL-1 $\beta$, and IL-6, quercetin also promotes the secretion of anti-inflammatory cytokines such as IL-10 (31).
The excellent anti-oxidant activity of quercetin is mainly exerted via effects on the activity of glutathione, enzymes, and reactive oxygen species (ROS), and regulating signal transduction pathways such as heme oxygenase 1 /nuclear factor erythroid 2-related factor (Nrf2), mitogen-activated protein kinase, toll-like receptor 4/phosphatidylinositol-3kinase, and 5' adenosine monophosphate-activated protein kinase (32). Quercetin can reduce high-valent iron, thereby inhibiting lipid oxidation and quenching ROS, assisting in suppressing inflammation and preventing related diseases (33). Kalantari et al. (34) reported that quercetin significantly alleviated liver damage in mice by suppressing free radicals and upregulating the levels of antioxidant enzymes including glutathione peroxidase, superoxide dismutase, and catalase. In another study, a quercetin-coated nanocellulose matrix had strong antioxidant activity (35). Bai et al. (36) formulated composite films of carboxymethyl chitosan-quercetin that exhibited anti-oxidant capacity in a sustained manner in aqueous and lipid foods. Lastly, it has been reported that quercetin has neuroprotective effects and anti-cancer activity.

Quercetin has undergone clinical trials, and to date no significant toxicity or side effects have been observed in humans. Inhibitory effects of quercetin on inflammation have been observed in clinical studies, as it has the capacity to suppress multiple types of cancer (37). More clinical studies should be conducted to confirm the effects of quercetin on autoimmune diseases, and better characterize the potential mechanisms of these beneficial effects.

\section{QUERCETIN AND AUTOIMMUNE DISEASES}

\section{Rheumatoid Arthritis}

Rheumatoid arthritis (RA) is a systemic chronic autoinflammatory disease. The main clinical symptoms are synovitis and progressive destruction of multiple joints, which can lead to joint deformity and dysfunction. It can also cause multi-system damage, and in severe cases premature death. In a randomized, double-blind, placebo-controlled clinical study, quercetin significantly alleviated morning stiffness and pain in RA patients (38). In that study, DAS 28 and HAQ scores of RA patients in a quercetin group where lower than those in a placebo group. In in vivo studies (39-41), quercetin has reduced arthritic scores and improved symptoms significantly in RA mice via inhibiting neutrophil infiltration and reducing levels of pro-inflammatory cytokines such as interferon $\gamma$, TNF- $\alpha$, monocyte chemotactic protein 1 , IL-6, and IL-17. It also inhibited neutrophil extracellular trap formation by suppressing autophagy (42). In another study, in a murine arthritis model, quercetin treatment reduced IL-1 $\beta$ and TNF- $\alpha$ release, and inhibited levels of pre-pro-endothelin 1 and cyclooxygenase 2 mRNA by inhibiting the activation of NF- $\mathrm{KB}$ and heme oxygenase 1/Nrf2 signaling (43). Yang et al. (44) found that quercetin attenuated collagen-induced arthritis by regulating the Th17/Treg balance, upregulating the expression of heme oxygenase 1 , and inhibiting the activation of NLRP3 
inflammasome. Saccol et al. (45) reported that quercetin protected against joint damage by reversing the deleterious effects of complete Freund's adjuvant-induced rat arthritis. In that study, the protective effects were closely associated with reductions in aspartate aminotransferase, ROS, and thiobarbituric acid-reactive substances, an increase in catalase activity, and reductions in DNA damage and fragmentation of double-strand DNA.

The aberrant migration, proliferation, and invasion of fibroblast-like synoviocytes (FLSs) are considered to be major parts of the etiopathogenesis of RA. In vitro, quercetin significantly inhibited the migration and invasion of FLSs, and reduced the levels of F-actin. It also increased the level of miR146a, but inhibited the expression of GATA transcription factor 6 in FLSs (46). Kim et al. (47) reported that quercetin reduced this osteoclast differentiation when $\mathrm{CD}^{+} 4^{+}$monocytes were cultured with IL-17-prestimulated RA-FLSs or Th17 cells, and it reduced IL-17-induced receptor activator of nuclear factor kappa-B ligand (RANKL) levels in RA-FLSs, and inhibited the activation of the mammalian target of rapamycin. Many studies indicate that quercetin can inhibit the generation of osteoclastlike cells, bone resorption depression, and F-actin ring formation in RAW264.7 cells, bone-marrow macrophages, and human peripheral-blood mononuclear cells pre-treated with RANKL or macrophage colony-stimulating factor (48-52). These results suggest that quercetin may play a role in protection against arthritic bone destruction.

RA affects extra-articular tissues and organs, including blood vessels, the nervous and gastrointestinal systems, heart, lung, and kidneys. In an adjuvant-induced arthritis model established in rats, Piovezana Bossolani et al. (53) detected neurodegenerative effects on the enteric nervous system of the jejunum, and inflammation in the intestinal mucosa. Treatment with quercetin alone reversed the aforementioned neurodegenerative effects to an extent, possibly due to its anti-inflammatory, antioxidant, anti-arthritic, and neuroprotective capacities.

\section{Inflammatory Bowel Disease}

Inflammatory bowel disease (IBD) encompasses a group of complicated and multifactorial polygenic conditions, mainly comprising ulcerative colitis and Crohn's disease. It is characterized by chronic, progressive, and relapsing inflammation in the gastrointestinal tract, which increases the risk of colitis-associated cancer. Quercetin has been shown to be a potent anti-inflammatory compound in a variety of in vitro and in vivo bioassay models, but oral quercetin has not exhibited the desired effects in a colitis model $(54,55)$. This could be partly because it is absorbed in the upper gastrointestinal tract and thus does not reach the lower gastrointestinal tract. The rapid metabolism of quercetin is disadvantageous with respect to treating IBD, unless the flavonoid is introduced in its glycosylated form, the most common of which is the compound rutin. Only approximately $10 \%$ of orally ingested rutin is generally able to pass epithelial cells, the rest is converted to sulphate and glucuronide derivatives by the gut microbiota to generate quercetin (56). Although rutin may have direct effects in the treatment of IBD, it is reasonable to surmise that its pharmacological activity and effects are exerted via conversion to the bioactive aglycone, quercetin (57). Rutin is hydrolyzed by the gut bacteria in the colon to synthesize quercetin, so it may act as a prodrug with quercetin being the active component. Rutin has been shown to have potent effects in vivo (58).

In a trinitrobenzenesulfonic acid-induced inflammatory colitis rat model, oral administration of 10 or $25 \mathrm{mg} / \mathrm{kg}$ of rutin reduced colonic damage (59). In a dextran sulfate sodium (DSS)-induced colitis mouse model, rutin had a positive role in controlling colonic inflammation and disease progression, as well as in the reduction of nitric oxide, iNOS, cyclooxygenase 2, and prostaglandin E2 (60). Mascaraque et al. (61) reported that rutin (57 mg/kg/day orally) significantly improved CD $4^{+} \mathrm{CD}_{2} 2 \mathrm{~L}^{+} \mathrm{T}$ cell transfer colitis in mice, but quercetin had no obvious effects. Rutin has also exhibited promising results in the rat model of acetic acid-induced colitis, in which pre-treatment (25 and 100 $\mathrm{mg} / \mathrm{kg}$ ) resulted in significant improvements in inflammatory indicators (62). In recent studies, quercetin alleviated DSSinduced colitis by strengthening intestinal integrity and liver antioxidant capacity, regulated ERK1/2-FKBP and RXR-STAT3 pathways (63), increased levels of glutathione in serum, and inhibited oxidative stress in a Caco- 2 cell model induced by $\mathrm{H}_{2} \mathrm{O}_{2}$ (64). Dietary supplementation with $30 \mathrm{mg} / \mathrm{kg}$ of quercetin exerts therapeutic effects in a Citrobacter rodentium-induced colitis model in C57BL/6 mice, in part due to its ability to attenuate pro-inflammatory cytokines and regulate gut microbiota (65). Ju et al. (66) reported that quercetin could ameliorate DSS-induced colitis, and speculated that this was most likely due to modulating the anti-inflammatory effects of macrophages via the heme oxygenase 1-dependent pathway.

Some evidence suggests that glycosylation of quercetin as demonstrated by rutin is an important structural feature of flavonoids with respect to their efficacy against IBD. The deglycosylation of flavonoids in the small intestine is induced by epithelial $\beta$-glucosidases and colonic microflora, resulting in the production of bioactive aglycones such as quercetin (67). Quercetin and its glycosides-which are common in the blood in conjugated products-need be designed such that postabsorption they release active quercetin with a specific pattern in the colon. Several experiments of formulations have been conducted in recent years to address this problem and enhance the pharmacological efficacy of quercetin. The oral administration of quercetin-loaded microcapsules generated with a pectin/casein polymer attenuated macroscopic damage and edema, reduced neutrophil recruitment, and reduced levels of IL-1 $\beta$ and IL-33 in the colon tissues of mice with acetic acidinduced colitis (68). Shen et al. (69) designed a dual-responsive prodrug micelle consisting of quercetin and the biocompatible glycol chitosan, which tended to accumulate in sites of intestinal inflammation, and exhibited better therapeutic efficacy than the free drugs quercetin and mesalazine in a mouse colitis model.

Overall, available evidence suggests the efficacy of orally ingested glycosylated forms of quercetin such as rutin, or quercetin delivered via drug carriers for IBS. Sulfasalazine, a common aminosalicylate drug, also functions via interaction with colonic microbiota resulting in the release of active 
moieties at the IBD site. Unfortunately, however, the nonpharmacologically active fragment of sulfadiazine that is cleaved during this process has systemic side effects. The main drugs currently used to treat IBD such as 5-aminosalicylates, corticosteroids, immune-modifying agents, and biologic agents have exhibited disadvantages including loss of efficacy, substantial costs, and unavailability of formulations designed for oral administration. Quercetin is one of the most abundant natural flavonoids, and has promising therapeutic potential for the treatment of IBD. Oral rutin leading to the release of the active biomolecule quercetin at the site of inflammation may be an effective therapy for IBD.

\section{Multiple Sclerosis}

Multiple sclerosis (MS) is an autoimmune inflammatory disease of the central nervous system characterized by extensive demyelination and neurodegeneration due to glia activation, oligodendrocyte death, and axon depletion. In an in vitro study conducted using peripheral blood mononuclear cells from MS patients, treatment with quercetin reduced their proliferation, and modulated levels of IL-1 $\beta$, matrix metalloproteinase 9, and TNF- $\alpha$ in cell culture supernatants (70). Chronic microglia activation can result in the production of inflammatory and neurotoxic mediators including nitric oxide, iNOS, and ROS, which are closely associated with the pathogenesis and development of MS (71). The compound 3'-O-(3chloropivaloyl) quercetin reduced the expression of iNOS in lipopolysaccharide-activated BV-2 microglia and inhibited the activation of NF- $\mathrm{KB}$ (72). In a mouse study utilizing experimental allergic encephalomyelitis, a Th1 cell-mediated inflammatory demyelinating disorder that is the most commonly used autoimmune model of MS, treatment with quercetin inhibited the IL-12-induced activation of JAK2, TYK2, STAT3, and STAT4, as well as Th1 differentiation (73). Mast cells are involved in inflammatory processes and allergic responses in which immunologic stimulation causes the production of inflammatory mediators. It has been suggested that mast cells are the immune gate of the brain, and are likely associated with neuropathologic processes including MS (74). Quercetin has been associated with reductions in the release of tryptase and IL-6, and inhibition of histidine decarboxylase mRNA from human mast cells (75). Quercetin may be useful in the complementary treatment for MS.

\section{Other Autoimmune Diseases}

Systemic lupus erythematosus is a disorder characterized by immune-mediated inflammation and over-production of autoantibodies. Quercitrin attenuated the symptoms of lupus nephritis in a systemic lupus erythematosus mouse model, partly via inhibition of $\mathrm{CD}^{+} \mathrm{T}$ cell activation and inflammatory reactions by macrophages (76). Graves' disease is the most common autoimmune thyroid disorder associated with hyperthyroidism. Quercetin can reportedly inhibit DNA damage by modulating oxidative stress in lymphocytes from Graves' disease patients in vitro (77). Atopic dermatitis is an autoimmune and inflammatory skin disease characterized by skin lesions exhibiting infiltration by mast cells, eosinophils, and macrophages. In a mouse model of atopic dermatitis, treatment with quercetin reduced the concentrations of TNF- $\alpha$, CCL17, IL4, IL-6, and IFN- $\gamma$ in skin tissues (78), downregulated HMGB1, RAGE, p-NF- $\kappa \mathrm{B}$, and ERK1/2 levels, and upregulated Nrf2 $(79,80)$.

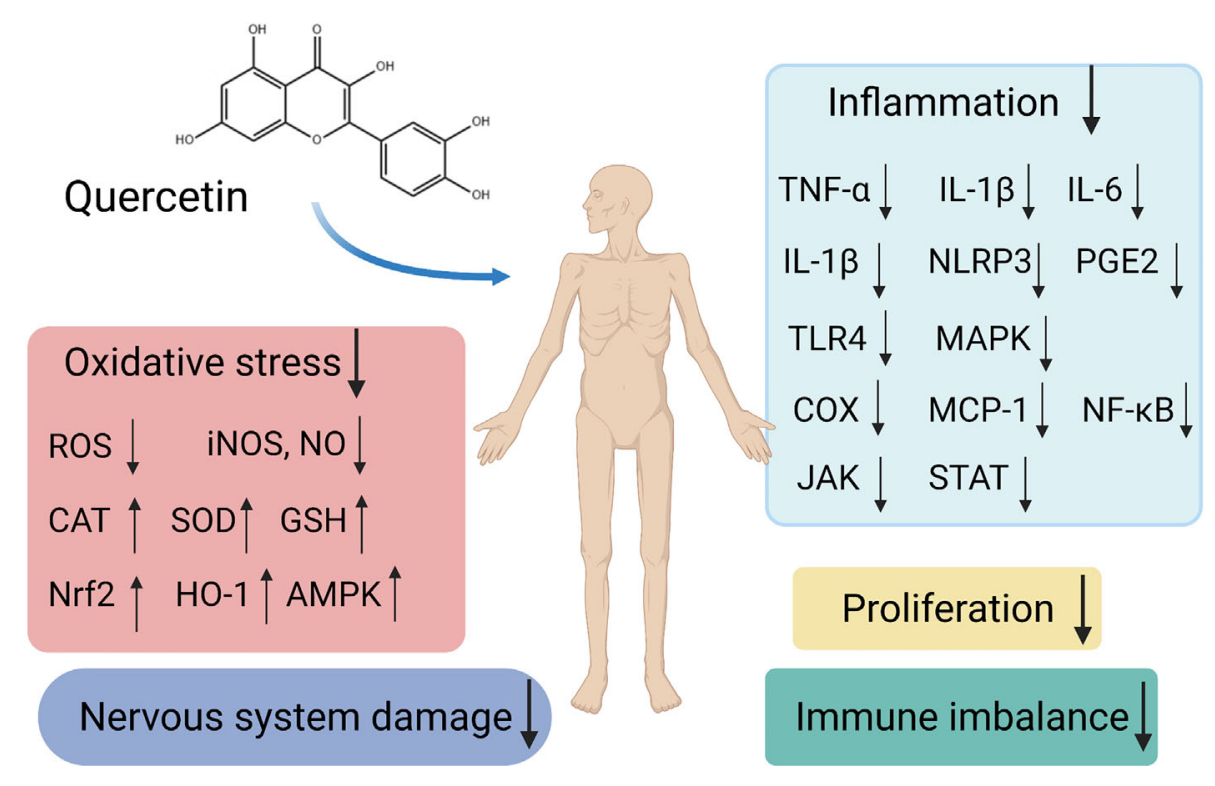

FIGURE 1 | Schematic representation of different signaling pathways and targets by quercetin as a potential therapeutic strategy in autoimmune diseases. 


\section{CONCLUSION}

Autoimmune diseases are systemic conditions that are difficult to cure, and patients often require long-term treatment. The development and pathogenesis of autoimmune diseases involve multiple associations and interacting factors. The diversity and complexity of associations between the components involved are likely to limit the effects of therapies, and contribute to adverse side effects. Quercetin possesses anti-inflammatory, anti-oxidant, neuroprotective, and anti-allergic activities, as well as the capacity to interact with multiple molecules and targets (Figure 1). Moreover, treatments involving appreciable doses of quercetin have evidently been low-toxic or non-toxic. Quercetin will be expected to become a potential opportunity and supplement for the treatment and prevention of autoimmune diseases. However, it is particularly important since there is no evidence so far that quercetin could reduce the morbidity and mortality of autoimmune diseases. The direct effects of quercetin on immune imbalance in patients are still unconfirmed. Further clinical studies are still lacking, most of the underlying mechanisms have been reported in animal models and need to be demonstrated for their pharmacological application. It is necessary to study it thoroughly with regard to high doses in order to detect possible undesirable side effects. Only well-designed randomized controlled trials with large sample sizes will reveal the biosafety and efficacy of these currently experimental applications. Due to its poor aqueous solubility, high metabolic rate, poor oral bioavailability and

\section{REFERENCES}

1. Cao F, Hu LQ, Yao SR, Hu Y, Wang DG, Fan YG, et al. P2X7 Receptor: A Potential Therapeutic Target for Autoimmune Diseases. Autoimmun Rev (2019) 18:767-77. doi: 10.1016/j.autrev.2019.06.009

2. Zhao CN, Xu Z, Wu GC, Mao YM, Liu LN, Qian W, et al. Emerging Role of Air Pollution in Autoimmune Diseases. Autoimmun Rev (2019) 18:607-14. doi: 10.1016/j.autrev.2018.12.010

3. Shukla SK, Singh G, Ahmad S, Pant P. Infections, Genetic and Environmental Factors in Pathogenesis of Autoimmune Thyroid Diseases. Microbial Pathogen (2018) 116:279-88. doi: 10.1016/j.micpath.2018.01.004

4. Babaei F, Mirzababaei M, Nassiri-Asl M. Quercetin in Food: Possible Mechanisms of Its Effect on Memory. J Food Sci (2018) 83:2280-7. doi: 10.1111/1750-3841.14317

5. Polerà N, Badolato M, Perri F, Carullo G, Aiello F. Quercetin and its Natural Sources in Wound Healing Management. Curr Med Chem (2019) 26:5825-48. doi: 10.2174/0929867325666180713150626

6. Yang D, Wang T, Long M, Li P. Quercetin: Its Main Pharmacological Activity and Potential Application in Clinical Medicine. Oxid Med Cell Longevity (2020) 2020:8825387. doi: 10.1155/2020/8825387

7. Shabbir U, Rubab M, Daliri EB, Chelliah R, Javed A, Oh DH. Curcumin, Quercetin, Catechins and Metabolic Diseases: The Role of Gut Microbiota. Nutrients (2021) 13(1):206. doi: 10.3390/nu13010206

8. Darband SG, Kaviani M, Yousefi B, Sadighparvar S, Pakdel FG, Attari JA, et al. Quercetin: A Functional Dietary Flavonoid With Potential ChemoPreventive Properties in Colorectal Cancer. J Cell Physiol (2018) 233:6544-60. doi: $10.1002 /$ jcp. 26595

9. Oboh G, Ademosun AO, Ogunsuyi OB. Quercetin and Its Role in Chronic Diseases. Adv Exp Med Biol (2016) 929:377-87. doi: 10.1007/978-3-31941342-6_17

10. Dhiman P, Malik N, Sobarzo-Sánchez E, Uriarte E, Khatkar A. Quercetin and Related Chromenone Derivatives as Monoamine Oxidase Inhibitors: absorption, and rapid body clearance, its application is limited. A better knowledge about the pharmacodynamics, pharmacokinetics, and enhanced bioavailability of quercetin are also necessary. In view of future directions and priorities, we suggest some protocol recommendations for future studies. Scholars can explore different routes of administration under the different medical conditions of different diseases such as the local injection for anti-inflammatory effects in RA. In in vitro studies, three-dimensional co-cultures of different cells that are responsible for a disease can mimic the microenvironment. For instance, fibroblast synovial cells, osteoblasts, and osteoclasts can be used in the studies of RA, and intestinal epithelial cells and fibroblasts can be adopted in IBD studies. Finally, it is valuable to increase the solubility, bioavailability, and target specificity of quercetin inside the human body. Investigating structurally relevant compounds of quercetin presents a novel subject for further experiments.

\section{AUTHOR CONTRIBUTIONS}

PS wrote the first draft of the manuscript. All authors contributed to the article and approved the submitted version.

\section{ACKNOWLEDGMENTS}

Figure 1 was created with BioRender.com.
Targeting Neurological and Mental Disorders. Mol (Basel Switzerland) (2019) 24(3):418. doi: 10.3390/molecules24030418

11. Huang YY, Wang ZH, Deng LH, Wang H, Zheng Q. Oral Administration of Quercetin or Its Derivatives Inhibit Bone Loss in Animal Model of Osteoporosis. Oxid Med Cell Longevity (2020) 2020:6080597. doi: 10.1155/ 2020/6080597

12. Dajas F. Life or Death: Neuroprotective and Anticancer Effects of Quercetin. J ethnopharmacol (2012) 143:383-96. doi: 10.1016/j.jep.2012.07.005

13. Patel RV, Mistry BM, Shinde SK, Syed R, Singh V, Shin HS. Therapeutic Potential of Quercetin as a Cardiovascular Agent. Eur J Med Chem (2018) 155:889-904. doi: 10.1016/j.ejmech.2018.06.053

14. Manach C, Scalbert A, Morand C, Rémésy C, Jiménez L. Polyphenols: Food Sources and Bioavailability. Am J Clin Nutr (2004) 79:727-47. doi: 10.1093/ ajcn/79.5.727

15. Murota K, Terao J. Antioxidative Flavonoid Quercetin: Implication of its Intestinal Absorption and Metabolism. Arch Biochem Biophys (2003) 417:127. doi: $10.1016 / \mathrm{s} 0003-9861<(>03<)>00284-4$

16. Williamson G, Barron D, Shimoi K, Terao J. In Vitro Biological Properties of Flavonoid Conjugates Found In Vivo. Free Radical Res (2005) 39:457-69. doi: 10.1080/10715760500053610

17. Bischoff SC. Quercetin: Potentials in the Prevention and Therapy of Disease. Curr Opin Clin Nutr Metab Care (2008) 11:733-40. doi: 10.1097/ MCO.0b013e32831394b8

18. Andres S, Pevny S, Ziegenhagen R, Bakhiya N, Schäfer B, Hirsch-Ernst KI, et al. Safety Aspects of the Use of Quercetin as a Dietary Supplement. Mol Nutr Food Res (2018) 62(1). doi: 10.1002/mnfr.201700447

19. Santangelo R, Silvestrini A, Mancuso C. Ginsenosides, Catechins, Quercetin and Gut Microbiota: Current Evidence of Challenging Interactions. Food Chem Toxicol An Int J Published Br Ind Biol Res Assoc (2019) 123:42-9. doi: $10.1016 /$ j.fct.2018.10.042

20. Tang SM, Deng XT, Zhou J, Li QP, Ge XX, Miao L. Pharmacological Basis and New Insights of Quercetin Action in Respect to Its Anti-Cancer Effects. 
Biomed Pharmacother = Biomedecine Pharmacotherapie (2020) 121:109604. doi: 10.1016/j.biopha.2019.109604

21. Najmanová I, Pourová J, Vopršalová $\mathrm{M}$, Pilařová $\mathrm{V}$, Semecký V, Nováková L, et al. Flavonoid Metabolite 3-(3-Hydroxyphenyl)Propionic Acid Formed by Human Microflora Decreases Arterial Blood Pressure in Rats. Mol Nutr Food Res (2016) 60:981-91. doi: 10.1002/mnfr.201500761

22. Dower JI, Geleijnse JM, Gijsbers L, Schalkwijk C, Kromhout D, Hollman PC. Supplementation of the Pure Flavonoids Epicatechin and Quercetin Affects Some Biomarkers of Endothelial Dysfunction and Inflammation in (Pre) Hypertensive Adults: A Randomized Double-Blind, Placebo-Controlled, Crossover Trial. J Nutr (2015) 145:1459-63. doi: 10.3945/jn.115.211888

23. Murakami A, Ashida H, Terao J. Multitargeted Cancer Prevention by Quercetin. Cancer Lett (2008) 269:315-25. doi: 10.1016/j.canlet.2008.03.046

24. Carullo G, Cappello AR, Frattaruolo L, Badolato M, Armentano B, Aiello F. Quercetin and Derivatives: Useful Tools in Inflammation and Pain Management. Future Med Chem (2017) 9:79-93. doi: 10.4155/fmc-2016-0186

25. Li G, Shen X, Wei Y, Si X, Deng X, Wang J. Quercetin Reduces Streptococcus Suis Virulence by Inhibiting Suilysin Activity and Inflammation. Int Immunopharmacol (2019) 69:71-8. doi: 10.1016/j.intimp.2019.01.017

26. Ahmed OM, Mohamed T, Moustafa H, Hamdy H, Ahmed RR, Aboud E. Quercetin and Low Level Laser Therapy Promote Wound Healing Process in Diabetic Rats Via Structural Reorganization and Modulatory Effects on Inflammation and Oxidative Stress. Biomed Pharmacother $=$ Biomedecine Pharmacotherapie (2018) 101:58-73. doi: 10.1016/j.biopha.2018.02.040

27. Okoko T, Oruambo IF. Inhibitory Activity of Quercetin and its Metabolite on Lipopolysaccharide-Induced Activation of Macrophage U937 Cells. Food Chem Toxicol An Int J Published Br Ind Biol Res Assoc (2009) 47:809-12. doi: 10.1016/j.fct.2009.01.013

28. Carrasco-Pozo C, Castillo RL, Beltrán C, Miranda A, Fuentes J, Gotteland M. Molecular Mechanisms of Gastrointestinal Protection by Quercetin Against Indomethacin-Induced Damage: Role of NF- $\mathrm{kb}$ and Nrf2. J Nutr Biochem (2016) 27:289-98. doi: 10.1016/j.jnutbio.2015.09.016

29. Endale M, Park SC, Kim S, Kim SH, Yang Y, Cho JY, et al. Quercetin Disrupts Tyrosine-Phosphorylated Phosphatidylinositol 3-Kinase and Myeloid Differentiation factor-88 Association, and Inhibits MAPK/AP-1 and IKK/ NF-kb-Induced Inflammatory Mediators Production in RAW 264.7 Cells. Immunobiology (2013) 218:1452-67. doi: 10.1016/j.imbio.2013.04.019

30. Yang D, Liu X, Liu M, Chi H, Liu J, Han H. Protective Effects of Quercetin and Taraxasterol Against $\mathrm{H}<(>2<)>\mathrm{O}<(>2<)>$-induced Human Umbilical Vein Endothelial Cell Injury In Vitro. Exp Ther Med (2015) 10:1253-60. doi: $10.3892 / \mathrm{etm} .2015 .2713$

31. Seo MJ, Lee YJ, Hwang JH, Kim KJ, Lee BY. The Inhibitory Effects of Quercetin on Obesity and Obesity-Induced Inflammation by Regulation of MAPK Signaling. J Nutr Biochem (2015) 26:1308-16. doi: 10.1016/ j.jnutbio.2015.06.005

32. Xu D, Hu MJ, Wang YQ, Cui YL. Antioxidant Activities of Quercetin and Its Complexes for Medicinal Application. Mol (Basel Switzerland) (2019) 24 (6):1123. doi: 10.3390/molecules24061123

33. Xiao L, Luo G, Tang Y, Yao P. Quercetin and Iron Metabolism: What We Know and What We Need to Know. Food Chem Toxicol An Int J Published $\mathrm{Br}$ Ind Biol Res Assoc (2018) 114:190-203. doi: 10.1016/j.fct.2018.02.022

34. Kalantari H, Foruozandeh H, Khodayar MJ, Siahpoosh A, Saki N, Kheradmand P. Antioxidant and Hepatoprotective Effects of Capparis Spinosa L. Fractions and Quercetin on Tert-Butyl Hydroperoxide- Induced Acute Liver Damage in Mice. J Taditional Complementary Med (2018) 8:1207. doi: $10.1016 /$ j.jtcme.2017.04.010

35. Li X, Liu Y, Yu Y, Chen W, Liu Y, Yu H. Nanoformulations of Quercetin and Cellulose Nanofibers as Healthcare Supplements With Sustained Antioxidant Activity. Carbohydr Polymers (2019) 207:160-8. doi: 10.1016/j.carbpol.2018.11.084

36. Bai R, Zhang X, Yong H, Wang X, Liu Y, Liu J. Development and Characterization of Antioxidant Active Packaging and Intelligent $\mathrm{Al}(3$ + )-sensing Films Based on Carboxymethyl Chitosan and Quercetin. Int J Biol macromolecules (2019) 126:1074-84. doi: 10.1016/j.ijbiomac.2018.12.264

37. Rauf A, Imran M, Khan IA, Ur-Rehman M, Gilani SA, Mehmood Z, et al. Anticancer Potential of Quercetin: A Comprehensive Review. Phytother Res PTR (2018) 32:2109-30. doi: 10.1002/ptr.6155

38. Javadi F, Ahmadzadeh A, Eghtesadi S, Aryaeian N, Zabihiyeganeh M, Rahimi Foroushani A, et al. The Effect of Quercetin on Inflammatory Factors and
Clinical Symptoms in Women With Rheumatoid Arthritis: A Double-Blind, Randomized Controlled Trial. J Am Coll Nutr (2017) 36:9-15. doi: 10.1080/ 07315724.2016 .1140093

39. Guazelli CFS, Staurengo-Ferrari L, Zarpelon AC, Pinho-Ribeiro FA, RuizMiyazawa KW, Vicentini F, et al. Quercetin Attenuates Zymosan-Induced Arthritis in Mice. Biomede Pharmacother = Biomedecine Pharmacotherapie (2018) 102:175-84. doi: 10.1016/j.biopha.2018.03.057

40. Kawaguchi K, Kaneko M, Miyake R, Takimoto H, Kumazawa Y. Potent Inhibitory Effects of Quercetin on Inflammatory Responses of CollagenInduced Arthritis in Mice. Endocrine Metab Immune Disord Drug Targets (2019) 19:308-15. doi: 10.2174/1871530319666190206225034

41. Haleagrahara N, Miranda-Hernandez S, Alim MA, Hayes L, Bird G, Ketheesan N. Therapeutic Effect of Quercetin in Collagen-Induced Arthritis. Biomed Pharmacother $=$ Biomedecine Pharmacotherapie (2017) 90:38-46. doi: 10.1016/j.biopha.2017.03.026

42. Yuan K, Zhu Q, Lu Q, Jiang H, Zhu M, Li X, et al. Quercetin Alleviates Rheumatoid Arthritis by Inhibiting Neutrophil Inflammatory Activities. J Nutr Biochem (2020) 84:108454. doi: 10.1016/j.jnutbio.2020.108454

43. Borghi SM, Mizokami SS, Pinho-Ribeiro FA, Fattori V, Crespigio J, ClementeNapimoga JT, et al. The Flavonoid Quercetin Inhibits Titanium Dioxide $(\mathrm{TiO}<(>2<)>)$-induced Chronic Arthritis in Mice. J Nutr Biochem (2018) 53:81-95. doi: 10.1016/j.jnutbio.2017.10.010

44. Yang Y, Zhang X, Xu M, Wu X, Zhao F, Zhao C. Quercetin Attenuates Collagen-Induced Arthritis by Restoration of Th17/Treg Balance and Activation of Heme Oxygenase 1-Mediated Anti-Inflammatory Effect. Int Immunopharmacol (2018) 54:153-62. doi: 10.1016/j.intimp.2017.11.013

45. Saccol R, da Silveira KL, Manzoni AG, Abdalla FH, de Oliveira JS, Dornelles GL, et al. Antioxidant, Hepatoprotective, Genoprotective, and Cytoprotective Effects of Quercetin in a Murine Model of Arthritis. J Cell Biochem (2020) 121:2792-801. doi: 10.1002/jcb.29502

46. Zhao J, Chen B, Peng X, Wang C, Wang K, Han F, et al. Quercetin Suppresses Migration and Invasion by Targeting miR-146a/GATA6 Axis in FibroblastLike Synoviocytes of Rheumatoid Arthritis. Immunopharmacol Immunotoxicol (2020) 42:221-7. doi: 10.1080/08923973.2020.1742732

47. Kim HR, Kim BM, Won JY, Lee KA, Ko HM, Kang YS, et al. Quercetin, a Plant Polyphenol, Has Potential for the Prevention of Bone Destruction in Rheumatoid Arthritis. J Med Food (2019) 22:152-61. doi: 10.1089/ jmf.2018.4259

48. Siddiqui JA, Sharan K, Swarnkar G, Rawat P, Kumar M, Manickavasagam L, et al. Quercetin-6-C- $\beta$-D-glucopyranoside Isolated From Ulmus Wallichiana Planchon Is More Potent Than Quercetin in Inhibiting Osteoclastogenesis and Mitigating Ovariectomy-Induced Bone Loss in Rats. Menopause (New York NY) (2011) 18:198-207. doi: 10.1097/gme.0b013e3181e84e67

49. Zhang L, Tian Z, Li W, Wang X, Man Z, Sun S. Inhibitory Effect of Quercetin on Titanium Particle-Induced Endoplasmic Reticulum Stress (ERS)-Related Apoptosis and In Vivoosteolysis. Biosc Rep (2017) 37(4):BSR20170961. doi: $10.1042 / \mathrm{bsr} 20170961$

50. Ge YW, Feng K, Liu XL, Zhu ZA, Chen HF, Chang YY, et al. Quercetin Inhibits Macrophage Polarization Through the P-38 $\alpha / \beta$ Signalling Pathway and Regulates OPG/RANKL Balance in a Mouse Skull Model. J Cell Mol Med (2020) 24:3203-16. doi: $10.1111 /$ jcmm.14995

51. Tripathi G, Raja N, Yun HS. Effect of Direct Loading of Phytoestrogens Into the Calcium Phosphate Scaffold on Osteoporotic Bone Tissue Regeneration. J Mater Chem B (2015) 3:8694-703. doi: 10.1039/c5tb01574j

52. Yamaguchi M, Weitzmann MN. Quercetin, a Potent Suppressor of NF- $\kappa b$ and Smad Activation in Osteoblasts. Int J Mol Med (2011) 28:521-5. doi: 10.3892/ ijmm.2011.749

53. Piovezana Bossolani GD, Silva BT, Colombo Martins Perles JV, Lima MM, Vieira Frez FC, Garcia de Souza SR, et al. Rheumatoid Arthritis Induces Enteric Neurodegeneration and Jejunal Inflammation, and Quercetin Promotes Neuroprotective and Anti-Inflammatory Actions. Life Sci (2019) 238:116956. doi: 10.1016/j.lfs.2019.116956

54. Comalada M, Camuesco D, Sierra S, Ballester I, Xaus J, Gálvez J, et al. In Vivo Quercitrin Anti-Inflammatory Effect Involves Release of Quercetin, Which Inhibits Inflammation Through Down-Regulation of the NF-kappaB Pathway. Eur J Immunol (2005) 35:584-92. doi: 10.1002/eji.200425778

55. Kwon KH, Murakami A, Tanaka T, Ohigashi H. Dietary Rutin, But Not its Aglycone Quercetin, Ameliorates Dextran Sulfate Sodium-Induced Experimental 
Colitis in Mice: Attenuation of Pro-Inflammatory Gene Expression. Biochem Pharmacol (2005) 69:395-406. doi: 10.1016/j.bcp.2004.10.015

56. Habtemariam S, Lentini G. The Therapeutic Potential of Rutin for Diabetes: An Update. Mini Rev Med Chem (2015) 15:524-8. doi: 10.2174/ 138955751507150424103721

57. Habtemariam S, Belai A. Natural Therapies of the Inflammatory Bowel Disease: The Case of Rutin and its Aglycone, Quercetin. Mini Rev Med Chem (2018) 18:234-43. doi: 10.2174/1389557517666170120152417

58. Kim H, Kong H, Choi B, Yang Y, Kim Y, Lim MJ, et al. Metabolic and Pharmacological Properties of Rutin, a Dietary Quercetin Glycoside, for Treatment of Inflammatory Bowel Disease. Pharm Res (2005) 22:1499-509. doi: $10.1007 /$ s11095-005-6250-z

59. Cruz T, Gálvez J, Ocete MA, Crespo ME, Sánchez de Medina LHF, Zarzuelo A. Oral Administration of Rutoside can Ameliorate Inflammatory Bowel Disease in Rats. Life Sci (1998) 62:687-95. doi: 10.1016/s0024-3205<(>97<)>01164-8

60. Marín M, Giner RM, Ríos JL, Recio Mdel C. Protective Effect of Apocynin in a Mouse Model of Chemically-Induced Colitis. Planta Med (2013) 79:1392-400. doi: $10.1055 / \mathrm{s}-0033-1350710$

61. Mascaraque C, Aranda C, Ocón B, Monte MJ, Suárez MD, Zarzuelo A, et al. Rutin has Intestinal Antiinflammatory Effects in the CD4+ Cd62l+ T Cell Transfer Model of Colitis. Pharmacol Res (2014) 90:48-57. doi: 10.1016/ j.phrs.2014.09.005

62. Gálvez J, Cruz T, Crespo E, Ocete MA, Lorente MD, Sánchez de Medina F, et al. Rutoside as Mucosal Protective in Acetic Acid-Induced Rat Colitis. Planta Med (1997) 63:409-14. doi: 10.1055/s-2006-957723

63. Dong Y, Lei J, Zhang B. Dietary Quercetin Alleviated DSS-induced Colitis in Mice Through Several Possible Pathways by Transcriptome Analysis. Curr Pharm Biotechnol (2020) 21:1666-73. doi: 10.2174/1389201021666200711152726

64. Dong Y, Hou Q, Lei J, Wolf PG, Ayansola H, Zhang B. Quercetin Alleviates Intestinal Oxidative Damage Induced by $\mathrm{H}<(>2<)>\mathrm{O}<(>2<)>$ Via Modulation of GSH: in Vitro Screening and In Vivo Evaluation in a Colitis Model of Mice. ACS Omega (2020) 5:8334-46. doi: 10.1021/acsomega.0c00804

65. Lin R, Piao M, Song Y. Dietary Quercetin Increases Colonic Microbial Diversity and Attenuates Colitis Severity in Citrobacter Rodentium-Infected Mice. Front Microbiol (2019) 10:1092. doi: 10.3389/fmicb.2019.01092

66. Ju S, Ge Y, Li P, Tian X, Wang H, Zheng X, et al. Dietary Quercetin Ameliorates Experimental Colitis in Mouse by Remodeling the Function of Colonic Macrophages Via a Heme oxygenase-1-Dependent Pathway. Cell Cycle (Georgetown Tex) (2018) 17:53-63. doi: 10.1080/15384101.2017.1387701

67. Németh K, Plumb GW, Berrin JG, Juge N, Jacob R, Naim HY, et al. Deglycosylation by Small Intestinal Epithelial Cell Beta-Glucosidases Is a Critical Step in the Absorption and Metabolism of Dietary Flavonoid Glycosides in Humans. Eur J Nutr (2003) 42:29-42. doi: 10.1007/s00394-003-0397-3

68. Guazelli CF, Fattori V, Colombo BB, Georgetti SR, Vicentini FT, Casagrande R, et al. Quercetin-Loaded Microcapsules Ameliorate Experimental Colitis in Mice by Anti-Inflammatory and Antioxidant Mechanisms. J Nat Prod (2013) 76:200-8. doi: 10.1021/np300670w

69. Shen C, Zhao L, Du X, Tian J, Yuan Y, Jia M, et al. Smart Responsive Quercetin-Conjugated Glycol Chitosan Prodrug Micelles for Treatment of Inflammatory Bowel Diseases. Mol Pharmaceutics (2021) 18:1419-30. doi: 10.1021/acs.molpharmaceut.0c01245
70. Sternberg Z, Chadha K, Lieberman A, Hojnacki D, Drake A, Zamboni P, et al. Quercetin and Interferon-Beta Modulate Immune Response(s) in Peripheral Blood Mononuclear Cells Isolated From Multiple Sclerosis Patients. J Neuroimmunol (2008) 205:142-7. doi: 10.1016/j.jneuroim.2008.09.008

71. Voet S, Prinz M, van Loo G. Microglia in Central Nervous System Inflammation and Multiple Sclerosis Pathology. Trends Mol Med (2019) 25:112-23. doi: 10.1016/j.molmed.2018.11.005

72. Mrvová N, Škandík M, Kuniaková M, Račková L. Modulation of BV-2 Microglia Functions by Novel Quercetin Pivaloyl Ester. Neurochem Int (2015) 90:246-54. doi: 10.1016/j.neuint.2015.09.005

73. Muthian G, Bright JJ. Quercetin, a Flavonoid Phytoestrogen, Ameliorates Experimental Allergic Encephalomyelitis by Blocking IL-12 Signaling Through JAK-STAT Pathway in T Lymphocyte. J Clin Immunol (2004) 24:542-52. doi: 10.1023/B:JOCI.0000040925.55682.a5

74. Ribatti D, Tamma R, Annese T. Mast Cells and Angiogenesis in Multiple Sclerosis. Inflammation Res Off J Eur Histamine Res Soc (2020) 69:1103-10. doi: 10.1007/s00011-020-01394-2

75. Kempuraj D, Castellani ML, Petrarca C, Frydas S, Conti P, Theoharides TC, et al. Inhibitory Effect of Quercetin on Tryptase and Interleukin-6 Release, and Histidine Decarboxylase mRNA Transcription by Human Mast Cell-1 Cell Line. Clin Exp Med (2006) 6:150-6. doi: 10.1007/s10238-006-0114-7

76. Li W, Li H, Zhang M, Wang M, Zhong Y, Wu H, et al. Quercitrin Ameliorates the Development of Systemic Lupus Erythematosus-Like Disease in a Chronic Graft-Versus-Host Murine Model. Am J Physiol Renal Physiol (2016) 311: F217-26. doi: 10.1152/ajprenal.00249.2015

77. Tang XL, Liu XJ, Sun WM, Zhao J, Zheng RL. Oxidative Stress in Graves' Disease Patients and Antioxidant Protection Against Lymphocytes DNA Damage In Vitro. Die Pharmazie (2005) 60:696-700.

78. Hou DD, Zhang W, Gao YL, Sun YZ, Wang HX, Qi RQ, et al. Anti-Inflammatory Effects of Quercetin in a Mouse Model of MC903-Induced Atopic Dermatitis. Int Immunopharmacol (2019) 74:105676. doi: 10.1016/j.intimp.2019.105676

79. Karuppagounder V, Arumugam S, Thandavarayan RA, Pitchaimani V, Sreedhar R, Afrin R, et al. Modulation of HMGB1 Translocation and RAGE/Nfikb Cascade by Quercetin Treatment Mitigates Atopic Dermatitis in NC/Nga Transgenic Mice. Exp Dermatol (2015) 24:418-23. doi: 10.1111/exd.12685

80. Lee HN, Shin SA, Choo GS, Kim HJ, Park YS, Kim BS, et al. Anti -Inflammatory Effect of Quercetin and Galangin in LPS-Stimulated RAW264.7 Macrophages and DNCB-induced Atopic Dermatitis Animal Models. Int J Mol Med (2018) 41:888-98. doi: 10.3892/ijmm.2017.3296

Conflict of Interest: The authors declare that the research was conducted in the absence of any commercial or financial relationships that could be construed as a potential conflict of interest.

Copyright (c) 2021 Shen, Lin, Deng, Ba, Han, Chen, Qin, Huang and Tu. This is an open-access article distributed under the terms of the Creative Commons Attribution License (CC BY). The use, distribution or reproduction in other forums is permitted, provided the original author(s) and the copyright owner(s) are credited and that the original publication in this journal is cited, in accordance with accepted academic practice. No use, distribution or reproduction is permitted which does not comply with these terms. 